\section{Autophagy: a new regulator of development}

In response to different stresses, cells undergo autophagy, which involves the engulfment of parts of the cytoplasm inside double-membraned vesicles called autophagosomes. Autophagosomes subsequently fuse with lysosomes and their cytoplasmic content is degraded. This process has been shown to delay the onset of neurodegeneration in different animal models by clearing up neuronal protein aggregates. However, the question remained whether autophagy was involved in normal development - an idea supported by circumstantial evidence and by a recent study showing the involvement of autophagy in the clearance of dead cells in the early mouse embryo (Qu et al. Cell 128, 931-946; 2007). Cecconi and colleagues have now identified a new positive regulator of Beclin-1, a key effector of autophagosome formation that is expressed exclusively in the developing nervous system. Analysis of its function reveals the importance of autophagy during neuronal development (Fimia et al. Nature doi:10.1038/nature05925).

The authors identified Ambral (activating molecule in Beclin-1 regulating autophagy), using a gene-trap approach to find genes expressed in the developing nervous system, and whose knockout led to neural-tube defects and embryonic lethality. Neural tubes in Ambra1-deficient embryos exhibit both an excess of programmed cell death and an increase in cell proliferation, but overall neuronal specification is normal, indicating the importance of Ambral for cell survival and growth.

In a yeast two-hybrid screen, the authors found that Ambra-1 interacts with Beclin-1, a component of the PI(3) kinase Vps34 containing complex, which is known to regulate autophagosome formation in mammals. Further analysis in human fibroblasts showed that Ambra-1 associates with the Beclin1-Vps34 kinase containing complex, and that Ambra-1 and Beclin-1 colocalise to vesicular structures.

Downregulation of Ambral by small interfering RNA (siRNA) in human fibroblasts decreases the levels of autophagy induced by two known triggers of the process - nutrient deprivation and mTOR inhibition. This is associated with an increase in cell proliferation. In addition, Ambra1-null mouse embryonic fibroblasts (MEFs) also exhibit defects in autophagy. As is observed for Beclin-1 overexpression, high levels of Ambral elicit autophagy in the absence of any stimulus, and decrease

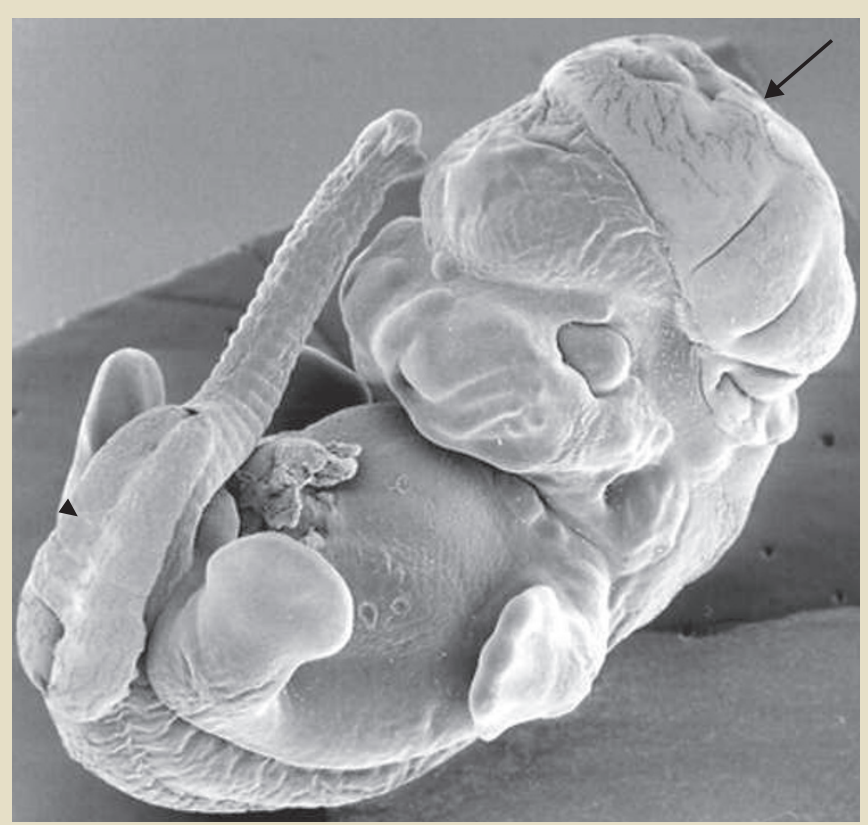

Electron scanning micrograph of an E12.5 Ambra1-mutant embryo. The mutant exhibits neural-tube defects (NTDs) detected as midbrain-hindbrain exencephaly (arrow) and/or spina bifida (arrowhead).

cell proliferation - an effect that requires the presence of Beclin-1. Furthermore, the authors showed that downregulation of Ambral impairs the interaction between Beclin-1 and the kinase Vps34.

These in vitro studies prompted the authors to examine the levels of autophagosome formation in the developing nervous system of wildtype and Ambra1-null mice. They found that autophagosomes were present in neuronal cells during their normal development, but almost absent in the brains of Ambra1-null mice. Previous studies have shown that impairment of autophagy in the adult nervous system leads to an accumulation of ubiquitinated proteins, and Ambra1-deficient embryos also exhibit high levels of ubiquitinated proteins in the brain.

Taken together, these findings indicate a role for autophagy in controlling protein turnover during neuronal development, and in regulating normal cell survival and proliferation. Whether the accumulation of ubiquitinated proteins is directly linked to defects in neuronal cell survival and proliferation remains to be determined.

NATHALIE LE BOT 